\title{
APROXIMACIÓN ANALÍTICA AL PRECIO DE UNA OPCIÓN AMERICANA: EVALUACIÓN A UN AÑO DE SU APARICIÓN EN MEXDER
}

\author{
Igor P. Rivera* \\ Centro de Investigación en Finanzas \\ Tecnológico de Monterrey, Campus Ciudad de México
}

(Recibido 5 de julio 2005, aceptado 8 de septiembre 2005)

\section{Resumen}

A poco más de un año del lanzamiento de las opciones en el MexDer, las opciones americanas de compra sobre acciones de América. Móvil serie L muestran en general un comportamiento muy similar al de las que serían sus equivalentes europeas; esto en virtud de que los dividendos decretados sobre la opción son muy pequeños con relación al precio de la acción. El presente estudio muestra la evidencia empírica de este comportamiento y describe como, en forma acotada, la fórmula de Black y Scholes pudo haberse utilizado como aproximación en el período de estudio.

\section{Abstract}

The aftermath on the first aniversary of launching American call options on America Movil L stock shows that thier price dynamics is quite similar to that of the corresponding European call options, because dividends on the stock are relatively small. This paper shows empirical evidence of such behavior and states that, in some way, Black-Scholes valuation formula could have been used to proxy the American call price within the sampled dates.

Clasificación JEL : G13, G29

Palabras clave: Valuación de opciones americanas, Mercados estandarizados de derivados

* Tecnológico de Monterrey, Campus Ciudad de México, Calle del Puente 222, Col. Ejidos de Huipulco. Tlalpan, c.p. 14380, México, D.F. Teléfono: +52 (55) 5483-2254. Correo electrónico: igrivera@itesm.mx

$\mathrm{El}$ autor desea agradecer a los directivos de ASIGNA, Compensación y Liquidación del Mercado Mexicano de Derivados, por los datos aportados para la realización de la presente investigación. Asimismo, se agradecen los valiosos comentarios de los dos dictaminadores anónimos. 


\section{Introducción}

A diferencia de las opciones europeas simples que, bajo el supuesto de normalidad de rendimientos del subyacente, son suceptibles de valuación por medio de la solución cerrada de Black y Scholes (1973), las opciones americanas no lo son, en virtud de que la distribución de las variaciones de precios del subyacente se vuelve discreta en un marco de tiempo finito. Esta peculiaridad ha sido atacada desde diferentes aristas, empezando por trabajos seminales como el de Merton (1973) en el que se sustituye el marco de tiempo finito por uno infinito, de tal suerte que se puede obtener una solución cerrada, y los de Roll (1977) y Geske (1979a) en los que se plantea el problema de valuación de opciones compuestas o anidadas, dado que la cualidad de ejercicio anticipado es equivalente al derecho (opción) de obtener un pago similar al de una opción europea en casi cualquier momento.

Sin embargo, las restricciones que imponen estos modelos han dirigido la investigación hacia aproximaciones y métodos numéricos. En lo que respecta a las aproximaciones numéricas, algunos de los trabajos que establecen una conexión con el trabajo de Merton, son el de McMillan (1986) que presenta una aproximación cuadrática para la valuación de opciones de venta sobre acciones que no pagan dividendos, Barone-Adesi y Whaley (1987) que utiliza también una aproximación cuadrática eficiente para opciones sobre físicos (commodities) y sus futuros, restringidos en ambos casos a tasas de interés y de acarreo constantes y distribuciones continuas del precio del subyacente. Cuando se mantiene la condición de distribución continua, los trabajos se basan en la idea propuesta por Geske de opciones compuestas; en esta línea destacan los trabajos de Geske (1979b), Whaley (1981 y 1986), Johnson (1983), Geske y Johnson (1984), Geske y Shastri (1985) y Blomeyer (1986).

En el campo de los métodos numéricos se han desarrollado técnicas basadas en árboles, lattices, esquemas de diferencias finitas y trayectorias simuladas para establecer los posibles valores finales de la función de pago y de ahí hacer un proceso recursivo de descuento para conocer el valor de la función de pago en cada nodo, para así poder calcular el valor presente de la opción. No obstante, este enfoque tiene algunas desventajas como son el aumento en la demanda de recursos computacionales con la dimensionalidad del problema, así como la necesidad de calibración de parámetros de magnitud de incrementos y decrementos, probabilidad de incremento y decremento y otros. En respuesta al problema de la dimensionalidad se ha recurrido a otras alternativas de simulación, dentro de las que destacan por su popularidad, las propuestas de Tilley (1993) y Barraquand y Martineau(1995). También, Broadie y Glasserman (1997) y Broadie, et al (1997) presentan una perspectiva amplia del uso de la metodología Monte Carlo en la valuación de opciones americanas. Algunos avances posteriores son resumidos por Fundia (2002). Adicionalmente, para una panorámica de los métodos de enrrejados refiérase a Briys et al. (1998).

Por otro lado, la negociación de productos derivados en México inició hace ya unas décadas, principalmente con forwards, swaps y warrants en operaciones realizadas generalmente sobre el mostrador. No obstante, no fue sino hasta 1997 cuando a iniciativa de la Bolsa Mexicana de Valores (BMV) y del Instituto de Depósito de Valores S.D. (Indeval) se conformó una bolsa organizada para la comercialización de derivados estandarizados. Así en 1998, inició 
operaciones MexDer, Mercado Mexicano de Derivados junto con su cámara de compensación, Asigna, Compensación y Liquidación. En su primer lustro de operaciones, el MexDer se posicionó como una bolsa de derivados medianamente activa, principalmente por la comercialización del futuro sobre la Tasa Interbancaria de Equilibrio (TIIE), y Asigna ha sido distinguida con la más alta calidad crediticia del país, por parte de tres reconocidas agencias calificadoras.

En 2004, el Mercado Mexicano de Derivados decidió ampliar el espectro de productos con el lanzamiento de opciones, con el apoyo tecnológico del MEFF (Mercado español de opciones) que ya tenía experiencia en la operación de este tipo de derivados. Las primeras opciones en listarse y comercializarse fucron las opciones europeas sobre el Índice de Precios y Cotizaciones (IPC) de la BMV y las opciones americanas sobre la acción de América Móvil serie L (AMXL). Por esta razón, es que el estudio se centra en el comportamiento de esta opción. americana, no obstante que al cabo de los meses, parece haber perdido el interés de los participantes del mercado, cediendo terreno a la opción del NAFTRAC2. En términos de volumen operado, a finales de 2004, las opciones europeas sobre el IPC casi alcanzaron el $90 \%$ mientras que las opciones americanas sobre AMXL no alcanzaron el $7 \%$ y el $4 \%$ restante correspondería a otras opciones americanas. En pocos meses, el auge de la opción sobre el NAFTRAC2 cambió esta perspectiva haciendo que en el acumulado de marzo de 2004 a junio de 2005 , las proporciones de volumen operado se volcaran hacia esta última clase de opción americana con más del $50 \%$, poco más del $45 \%$ para la opción europea del IPC y tan sólo 3\% para la opción americana sobre AMXL. De hecho en 2005, ya no se reportaron nuevas operaciones de la opción sobre AMXL.

\section{Justificación de la aproximación}

En la literatura es conocido que una opción americana de compra sobre una acción que no paga dividendos no ofrece incentivos para su ejercicio anticipado por lo que su valor es igual al de la opción europea con los mismos parámetros. Para revisar los fundamentos de esta equivalencia recuérdese que una opción americana de compra permite el ejercicio anticipado de los derechos de compra del subyaccnte (por ejemplo, una acción), a diferencia de la opción europea, que únicamente concede ejercerlos a su vencimiento. Esta concesión adicional tiene un valor en el mercado, pero no puede hacer que el contrato valga más que el subyacente sobre el que se tiene derecho de compra, por lo que en general se debe cumplir que el precio de una opción americana de compra esté acotado por:

$$
S_{0} \geq C_{0} \geq c_{0} \geq 0
$$

donde $C_{t}$ y $c_{t}$ denotan los precios al tiempo $t$ de la opción de compra de tipo americano y de tipo europeo, respectivamente. Además, considerando que el perfil de pago de la opción americana de compra, al vencimiento $T$ (suponiendo que no se ejerció anticipadamente) es $C_{T}=\max \left(S_{T}-X, 0\right)$, en cualquier tiempo $t$ se debe cumplir que:

$$
S_{t} \geq C_{t} \geq S_{t}-X \mathrm{e}^{-r(T-t)} \geq 0 .
$$

Suponga que se valuará la opción americana en el dinero sobre una acción que no paga dividendos, por medio de un árbol binomial de $N$ niveles como el 
propuesto por Cox, Ross y Rubinstein (1979) en el que:

$$
\begin{aligned}
u & =\mathrm{e}^{\sigma \sqrt{\tau}}, \\
d & =\mathrm{e}^{-\sigma \sqrt{\tau}}, \\
p & =\frac{\mathrm{e}^{r \tau}-d}{u-d}, \\
\tau & =\frac{T}{N-1} .
\end{aligned}
$$

En este contexto, la ausencia de dividendos implica que el árbol es recombinante. En un caso extremo en el que $\sigma \rightarrow 0, r \rightarrow 0$ y $N \geq 2$ tendríamos que los valores de la ecuación (1) serían:

$$
\begin{aligned}
& u \approx 1+\sigma \sqrt{T /(N-1)} \\
& d \approx 1-\sigma \sqrt{T /(N-1)}, \\
& p \rightarrow 0.5^{+} \\
& \tau=T / 2 .
\end{aligned}
$$

Además, se tiene que tanto $u$ como $d$ tienden a 1 . Por lo tanto el valor de la opción americana en $t=0$ puede expresarse como:

$$
C_{0} \approx S_{0}-X
$$

Pero cuando $r>0$, el sustraendo se hace menor y la diferencia aumenta, por lo que se debe cumplir que:

$$
C_{0}>S_{0}-X \mathrm{e}^{-r T},
$$

es decir, que en $t=0$ la tenencia de la opción y del dinero para comprar la acción al precio pactado de ejercicio, nos coloca en mejor situación que la tenencia de la acción misma. Este argumento debería bastar para demostrar que aun en un marco determinista, a menos que se decretaran dividendos en cantidad suficiente para compensar el valor de la opción en el tiempo (como consecuencia de $r>0$ ), no existirían incentivos para el ejercicio anticipado. Para efectos de esta demostración hay dos argumentos adicionales definitivos: primero, que se supone la inexistencia de dividendos, por lo que no hay cantidad tal que compense el valor de la opción en el tiempo, y, segundo, que el efecto de la volatilidad, $\sigma>0$, también aumenta el valor de la opción en el tiempo, lo que se puede ver de las definiciones en (1), ya que con $\sigma>0$, la probabilidad $p$ de que el subyacente crezca y que por ende el perfil de pagos termine más dentro de la región el dinero, aumenta por arriba de su cota inferior de 0.5.

En el Cuadro 1 se muestra que, en ausencia de dividendos, la valuación de la opción de compra (call) de tipo americano y de tipo europeo por medio de un árbol binomial es idéntica. Asimismo, la Gráfica 1 muestra que la valuación binomial tanto de la opción europea (línea sólida a cuadros) y americana (línea punteada a triángulos), convergen al valor calculado por la ecuación de Black y Scholes (línea sólo punteada); por ello, la aproximación por la técnica de 
variable de control (línea punteada a cruces), como la presentan Hull y White (1988) y Hull (2002), es idéntica al valor de la calculada por la fórmula cerrada.

Cuadro 1. Precios teóricos de opciones de compra.

\begin{tabular}{|c|c|c|c|c|}
\hline Niveles & $\begin{array}{c}\text { Europea } \\
\text { (B\&S) }\end{array}$ & $\begin{array}{c}\text { Europea } \\
\text { Binomial }\end{array}$ & $\begin{array}{c}\text { Americana } \\
\text { Binomial }\end{array}$ & $\begin{array}{c}\text { Americana } \\
\text { (CVT) }\end{array}$ \\
\hline 5 & 2.6250 & 2.6268 & 2.6268 & 2.6250 \\
10 & 2.6250 & 2.6532 & 2.6532 & 2.6250 \\
50 & 2.6250 & 2.6174 & 2.6174 & 2.6250 \\
100 & 2.6250 & 2.6285 & 2.6285 & 2.6250 \\
500 & 2.6250 & 2.6257 & 2.6257 & 2.6250 \\
\hline $\mathrm{S}=21$ & $\sigma=0.29$ & $\mathrm{r}=0.68$ & $\mathrm{X}=22$ & $\mathrm{~T}=1$ año \\
\hline \multicolumn{5}{c}{ B\&S = Valuación por Black \& Scholes } \\
\hline \multicolumn{5}{c}{ CVT Valuación por Técnica de Variable de Control } \\
\hline
\end{tabular}

Gráfica 1. Convergencia de los precios teóricos de opciones de compra, europea y americana.

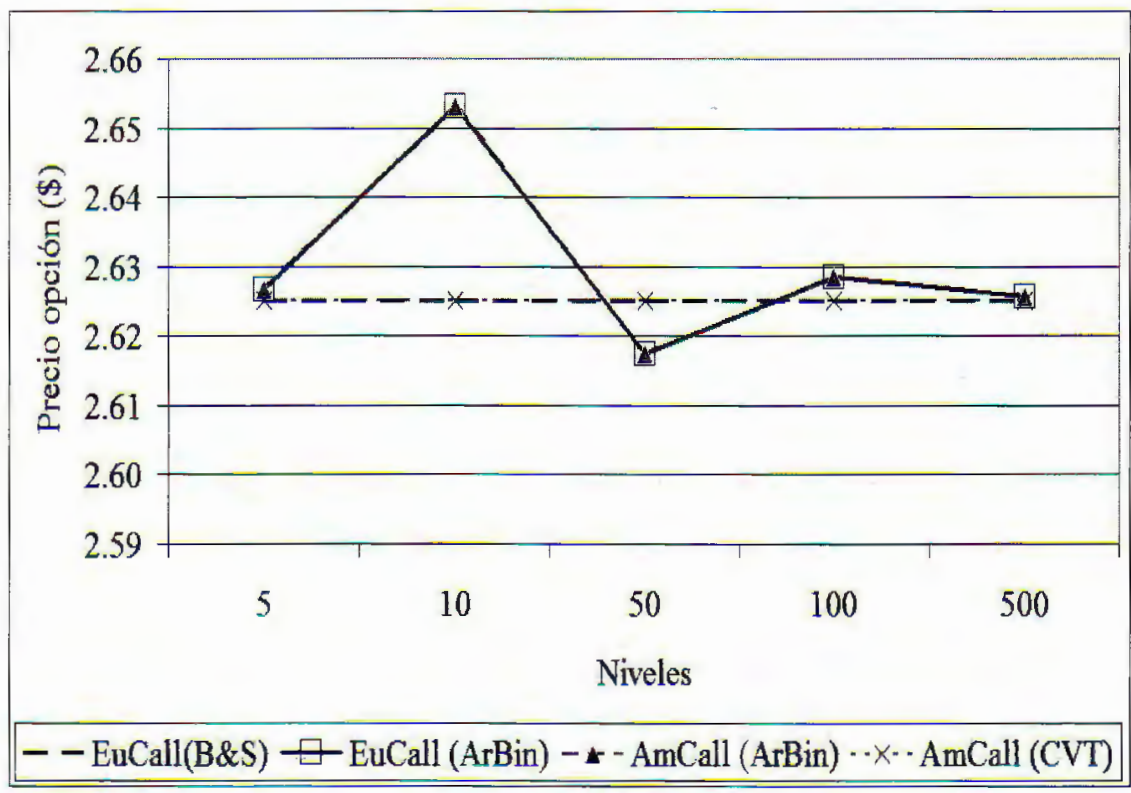

Por el contrario, en el caso de las opciones de venta (puts) sobre acciones que no pagan dividendos, la opción americana, valuada por medio de un árbol binomial, resulta más cara que la correspondiente opción europea, como se muestra en el Cuadro 2. Además, como se ilustra en la Gráfica 2, a mayor número de niveles se produce convergencia del precio binomial de la opción americana (punteada a triángulos), al valor calculado por variable de control (punteada a cruces), así como el precio binomial de la opción europea (sólida a cuadros), converge al valor de Black y Scholes (sólo punteada). 
Cuadro 2. Precios teóricos de opciones de venta.

\begin{tabular}{|c|c|c|c|c|}
\hline Niveles & $\begin{array}{c}\text { Europea } \\
\text { (B\&S) }\end{array}$ & $\begin{array}{c}\text { Europea } \\
\text { Binomial }\end{array}$ & $\begin{array}{c}\text { Americana } \\
\text { Binomial }\end{array}$ & $\begin{array}{c}\text { Americana } \\
\text { (CVT) }\end{array}$ \\
\hline 5 & 2.1787 & 2.1805 & 2.4263 & 2.4246 \\
10 & 2.1787 & 2.2069 & 2.4003 & 2.3721 \\
50 & 2.1787 & 2.1711 & 2.3916 & 2.3993 \\
100 & 2.1787 & 2.1822 & 2.3967 & 2.3932 \\
500 & 2.1787 & 2.1794 & 2.3939 & 2.3933 \\
\hline $\mathrm{S}=21$ & $\sigma=0.29$ & $\mathrm{r}=0.68$ & $\mathrm{X}=22$ & $\mathrm{~T}=1$ año \\
\hline \multicolumn{5}{c|}{ B\&S = Valuación por Black \& Scholes } \\
\hline \multicolumn{5}{c}{$=$ Valuación por Técnica de Variable de Control } \\
\hline
\end{tabular}

Gráfica 2. Divergencia entre los precios teóricos de opciones de venta, europea y americana.

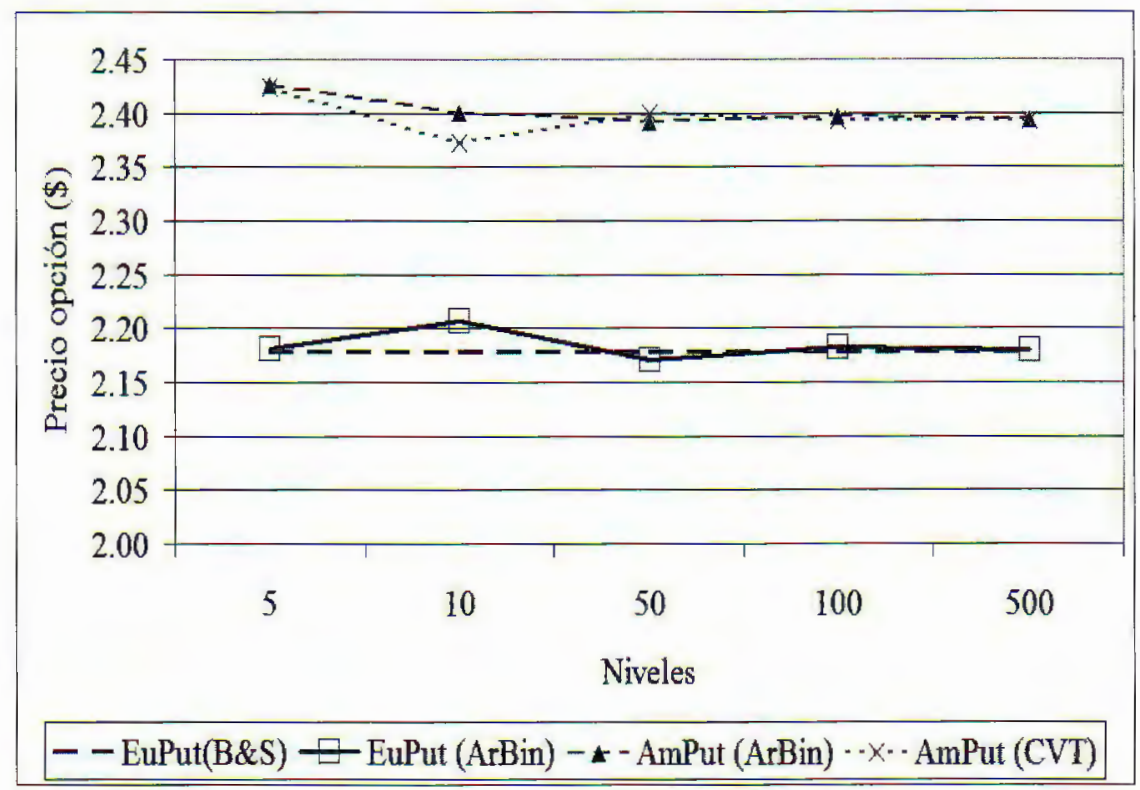

El Cuadro 3 resume la información obtenida de la sección de derechos de AMXL de "Economática" sobre los dividendos decretados para los tenedores de la acción en cuestión. Como se puede observar, la proporción de los dividendos con respecto al precio de la acción, hace suponer que el efecto de los dividendos sobre la valuación de la opción de compra puede ser despreciable, lo que hace posible aproximar el precio de la opción americana de compra por medio de la fórmula de Black y Scholes. 
Cuadro 3. Dividendos decretados como porcentaje del precio de la acción.

\begin{tabular}{|c|c|c|c|c|c|}
\hline Fecha & en $\$$ & $\begin{array}{c}\text { \% Precio } \\
\text { del día }\end{array}$ & $\begin{array}{c}\% \text { Prom. } \\
1 \text { mes }\end{array}$ & $\begin{array}{c}\text { \% Prom. } \\
6 \text { meses }\end{array}$ & $\begin{array}{c}\text { \% Prom. } \\
1 \text { año }\end{array}$ \\
\hline $25 / 06 / 2001$ & $\$ 0.0033333$ & $0.036 \%$ & $0.035 \%$ & ND & ND \\
$24 / 09 / 2001$ & $\$ 0.0033333$ & $0.050 \%$ & $0.047 \%$ & $0.039 \%$ & ND \\
$23 / 12 / 2001$ & $\$ 0.0033333$ & $0.038 \%$ & $0.040 \%$ & $0.042 \%$ & ND \\
$22 / 03 / 2002$ & $\$ 0.0033333$ & $0.036 \%$ & $0.038 \%$ & $0.042 \%$ & $0.040 \%$ \\
$24 / 06 / 2002$ & $\$ 0.0036667$ & $0.056 \%$ & $0.047 \%$ & $0.043 \%$ & $0.044 \%$ \\
$23 / 09 / 2002$ & $\$ 0.0036667$ & $0.055 \%$ & $0.053 \%$ & $0.049 \%$ & $0.047 \%$ \\
$20 / 12 / 2002$ & $\$ 0.0036667$ & $0.048 \%$ & $0.049 \%$ & $0.054 \%$ & $0.048 \%$ \\
$24 / 03 / 2003$ & $\$ 0.0036667$ & $0.050 \%$ & $0.049 \%$ & $0.051 \%$ & $0.049 \%$ \\
$23 / 06 / 2003$ & $\$ 0.005$ & $0.053 \%$ & $0.053 \%$ & $0.061 \%$ & $0.067 \%$ \\
$22 / 09 / 2003$ & $\$ 0.005$ & $0.039 \%$ & $0.039 \%$ & $0.049 \%$ & $0.057 \%$ \\
$21 / 12 / 2003$ & $\$ 0.005$ & $0.033 \%$ & $0.034 \%$ & $0.039 \%$ & $0.048 \%$ \\
$22 / 03 / 2004$ & $\$ 0.005$ & $0.025 \%$ & $0.025 \%$ & $0.031 \%$ & $0.038 \%$ \\
$21 / 06 / 2004$ & $\$ 0.01$ & $0.048 \%$ & $0.049 \%$ & $0.051 \%$ & $0.062 \%$ \\
$20 / 09 / 2004$ & $\$ 0.01$ & $0.045 \%$ & $0.049 \%$ & $0.049 \%$ & $0.055 \%$ \\
$20 / 12 / 2004$ & $\$ 0.01$ & $0.035 \%$ & $0.038 \%$ & $0.045 \%$ & $0.048 \%$ \\
$17 / 03 / 2005$ & $\$ 0.01$ & $0.033 \%$ & $0.032 \%$ & $0.037 \%$ & $0.042 \%$ \\
$22 / 06 / 2005$ & $\$ 0.0175$ & $0.055 \%$ & $0.056 \%$ & $0.059 \%$ & $0.067 \%$ \\
\hline
\end{tabular}

* Fecha de lanzamiento de las opciones en MexDer.

Fuente: Economática

En virtud de que el dividendo es muy pequeño con respecto a la acción, se planteó que el valor calculado por medio de la fórmula de Black y Scholes debía ser una buena aproximación al valor de mercado de la opción americana,

$$
\begin{aligned}
C & \approx S \Phi\left(d_{1}\right)-X \mathrm{e}^{-r(T-t)} \Phi\left(d_{2}\right) \\
d_{1} & =\frac{\ln \left(S_{0} / X\right)+\left(r+0.5 \sigma^{2}\right)(T-t)}{\sigma \sqrt{T-t}} \\
d_{2} & =d_{1}-\sigma \sqrt{T-t}
\end{aligned}
$$

dados los parámetros de precio de la acción, $S$, precio de ejercicio, $X$, tiempo al vencimiento, $T-t$, tasa de interés libre de riesgo, en este caso la TIIE, representada por la variable $r$, la volatilidad, $\sigma$, y donde $\Phi$ representa la función de distribución normal acumulada. El problema medular en este caso se torna sobre el valor de la volatilidad, ya que no es una variable observable; sin embargo, dado que se supone la factibilidad de uso de la fórmula de Black y Scholes para el cálculo de precios, también se puede utilizar para la determinación de la volatilidad implícita.

En este caso particular se supone que al listarse una nueva clase, se desconoce el comportamiento de la volatilidad por lo que se tomó como valor de referencia, la volatilidad anual del precio de la acción. Pero a partir del segundo 
día de listada la opción, se emplea como parámetro a la volatilidad implícita calculada sobre el precio realizado el día anterior; este supuesto sobre el comportamiento de la volatilidad es simplificador, pero es natural pensar que modelos econométricos de heteroscedasticidad condicional o modelos estocásticos de la dinámica de la volatilidad podrían mejorar la estimación, comparación que escapa al alcance de este breve estudio. El resto de los parámetros se suponen observables, y de hecho lo son, generalemente, con algunos minutos de retraso.

\section{Análisis de resultados}

Para poder mostrar la validez de los resultados obtenidos, se realizó una regresión lineal de los precios aproximados con respecto a los realizados:

$$
P_{\text {aprox }, t}=k+\dot{m} P_{\text {real }, t}+\epsilon_{t} .
$$

Si cumplen los supuestos establecidos, la regresión debe producir un recta de pendiente $m=1$ con ordenada al origen $k=0$. El Cuadro 4 muestra los resultados de la regresión de una serie para cada uno de los vencimientos, incluídos todos los precios de ejercicio. Como se puede observar, en todas las regresiones, el coeficiente de determinación, $R^{2}$, es satisfactorio, y los estimadores de los parámteros son como los esperados. En el caso particular de la ordenada al origen, la prueba $t$ no permite establecer que el estimador sea estadísticamente distinto de cero, situación que para los efectos de este trabajo se considera irrelevante dado que el valor esperado para dicho parámetro es precisamente cero.

Cuadro 4. Resultados de la regresión por vencimiento.

\begin{tabular}{|c|c|c|c|c|c|}
\hline \multicolumn{6}{|c|}{ Fechas de vencimiento } \\
\hline & $18 / 06 / 04$ & $17 / 09 / 04$ & $17 / 12 / 04$ & $18 / 03 / 05$ & $17 / 06 / 05$ \\
\hline$\hat{k}$ & 0.00456 & 0.00584 & 0.00748 & 0.00301 & 0.00443 \\
Estadístico $t$ & 1.41 & 1.90 & 2.23 & 0.92 & 1.10 \\
Probabilidad & $15.8 \%$ & $5.7 \%$ & $2.6 \%$ & $35.6 \%$ & $27.2 \%$ \\
\hline$\hat{m}$ & 0.99863 & 0.99586 & 0.99487 & 0.99709 & 0.99493 \\
Estadístico $t$ & 706.80 & 874.74 & 930.43 & $1,315.45$ & $1,121.07$ \\
Probabilidad & $0.0 \%$ & $0.0 \%$ & $0.0 \%$ & $0.0 \%$ & $0.0 \%$ \\
\hline$R^{2}$ & 0.99895 & 0.99848 & 0.99814 & 0.99877 & 0.99835 \\
Estadístico $F$ & $>10^{5}$ & $>10^{5}$ & $>10^{5}$ & $>10^{6}$ & $>10^{6}$ \\
Probabilidad & $0.0 \%$ & $0.0 \%$ & $0.0 \%$ & $0.0 \%$ & $0.0 \%$ \\
\hline
\end{tabular}

El Cuadro 5 resume los resultados de una regresión sobre todos los precios pronósticados, que en total suman 7,531 entre el 22 de marzo de 2004, fecha de lanzamiento de las opciones en MexDer, y el 17 de junio de 2005. El estimador de la ordenada al origen es cercano a cero, así como el estimador de la pendiente es cercano a uno, como se esperaba. No se hicieron otras pruebas de bondad de ajuste en virtud de que no se hacen comparaciones con otros modelos. 
También, se modificó una prueba empírica que originalmente planteó Whaley (1982) para comparar el bid-ask spread contra el promedio del error porcentual de diversos modelos de valuación. Dado que el promedio puede compensar valores negativos con positivos, en este caso mejor se realizó una medición sobre el valor absoluto de la desviación porcentual de los precios aproximados con respecto de los precios realizados. Para el conjunto de todos los precios, el el $90.3 \%$ de los precios presentan una desviación menor al $2 \%$, mientras que el 94.6\% de los precios estimados presentan una desviación menor al 5\%. En el caso del MexDer, el diferencial entre las posturas de compra y de venta (bid-ask spread) en opciones más líquidas oscila cerca del $4 \%$ por lo que al menos el $93 \%$ de los datos estimados, tendrían una desviación perfectamente admisible.

Cuadro 5. Resultados de la regresión general.

\begin{tabular}{|c|c|c|}
\hline \multicolumn{3}{|c|}{ Ordenada al origen, $k$} \\
\hline Estimador & Estadístico $t$ & Probabilidad \\
0.00550 & 3.41 & $0.1 \%$ \\
\hline \multicolumn{3}{|c|}{ Pendiente, $m$} \\
\hline Estimador & Estadístico $t$ & Probabilidad \\
0.99569 & $2,351.86$ & $0.0 \%$ \\
\hline Coef. determinación y prueba conjunta \\
\hline$R^{2}$ & Estadístico $F$ & Probabilidad \\
0.99864 & $>10^{6}$ & $0.0 \%$ \\
\hline
\end{tabular}

Cabe señalar que en ciertas situaciones en las que el precio realizado es muy cercano a cero, por ejemplo cuando la opción se encuentra fuera del dinero o bien si se encuentra al dinero y muy cerca de su vencimiento, el valor teórico puede ser exactamente igual a cero; en dichas situaciones, la desviación porcentual puede ser cercana o igual al 100\%, aunque en términos prácticos, la existencia de fricciones del mercado, hacen que la diferencia sea despreciable. Por ello, el análisis de desviaciones porcentuales se realizó con un filtrado que no considera aquellas desviaciones en las que el precio realizado de referencia es igual o menor a un cierto umbral, en este caso, de 0 (sin filtrado), 1 y 2 centavos de peso. El Cuadro 6 resume los resultados de dicho análisis.

Cuadro 6. Desviaciones porcentuales con respecto a precios realizados.

\begin{tabular}{|c|cccc|}
\hline Umbral & \multicolumn{4}{|c|}{ Valor crítico del bid-ask spread } \\
de filtrado & $2 \%$ & $3 \%$ & $4 \%$ & $5 \%$ \\
\hline $0 \phi$ & $90.3 \%$ & $92.2 \%$ & $93.4 \%$ & $94.6 \%$ \\
$1 \phi$ & $90.8 \%$ & $92.6 \%$ & $93.9 \%$ & $95.1 \%$ \\
$2 \varnothing$ & $91.0 \%$ & $92.9 \%$ & $94.1 \%$ & $95.3 \%$ \\
\hline
\end{tabular}

\section{Las limitaciones y posibles avenidas}

Para efectos de simplificar al estudio, no se ha especificado un modelo econométrico formal para la dinámica de la volatilidad $\mathrm{y}$, sencillamente, se supone que la volatilidad implícita del día anterior es un estimador de la volatilidad que se dará 
en el tiempo presente. Cabe enfatizar, que ni siquiera se pretende establecer que dicho estimador sea insesgado ni que los errores de la aproximación tienen una distribución específica. El hecho a establecer es el del comportamiento de la opción sobre AMXL como una opción europea, como consecuencia de que los dividendos decretados sobre el título subyacente son muy pequeños en relación al precio de la acción. En este caso, a menos que se pudiera garantizar el comportamiento suave de las series de volatilidad, puede ocurrir que los hechos de mercado produzcan volatilidades implícitas significativamente distintas a las de la tendencia de la serie. En dichos casos, el modelo producirá resultados menos precisos. No obstante, es de esperarse que tras algunas jornadas de operación, el modelo logre capturar la filtración para producir nuevamente resultados precisos. A manera de ejemplo, en la Gráfica 3 se muestran los precios realizado y aproximado, entre el 22 de junio y el 5 de agosto de 2004, de la opción de compra con vencimiento al 17 de septiembre del mismo año, con un precio de ejercicio de $\$ 24$ pesos. Dentro de esta muestra, el día 8 de julio se reportó un precio de $\$ 22$ centavos para la opción referida, pero al día siguiente el precio reportado fue de cero, precio que produjo un cálculo de volatilidad implicita de 0.06 para el 9 de julio, cuando el promedio de la volatilidad para las 2 semanas anteriores fue de 0.26 y el dato del día anterior de 0.19 . Con el dato de volatilidad implícita del 9 de julio, en el siguiente día hábil, 12 de julio, se produce un precio aproximado de cero, cuando el realizado para esta nueva fecha fue de 15 centavos. Durante algunos días subsecuentes se presentó un comportamiento igualmente errático en las series de precios y de volatilidad implícita, pero tan pronto se estabilizaron las mismas, la desviación de los precios aproximados con respecto a los realizados nuevamente vuelve a quedar en un rango aceptable.

Gráfica 3. Efectos de turbulencia. Precio realizado vs. Precio aproximado.

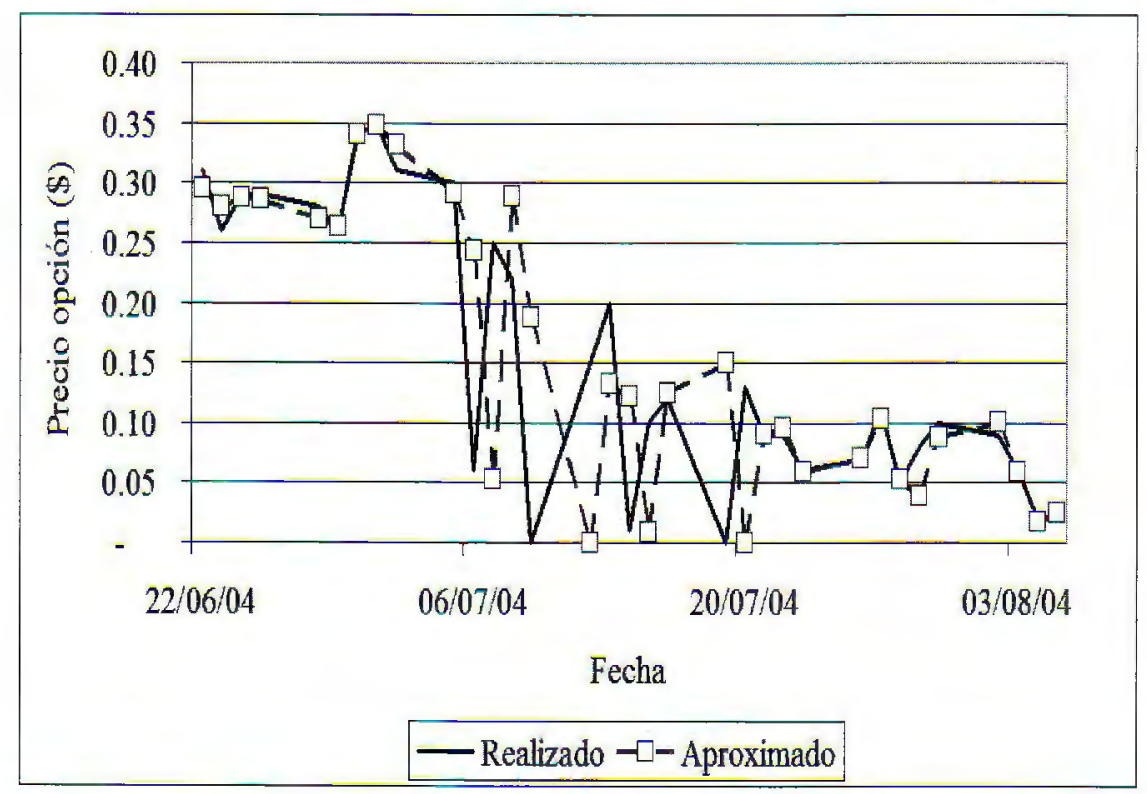


El presente trabajo se ha centrado en el estudio de la dinámica de la primera opción americana operada en el MexDer. Sin embargo, como en todos los sistemas que inician su operación, existe un lapso, dentro de la etapa de producción, en la que se precisan ajustes finos y se inicia un camino a través de la curva de aprendizaje. Hoy en día, la opción americana sobre AMXL no es la única, ni mucho menos es la más líquida. Por ello, otras investigaciones podrían dar seguimiento a lo que ocurre con otros productos listados, y que hoy por hoy son mucho más líquidos y tienen mayor profundidad de mercado. Asimismo, la parte del modelo que se enfoca en la volatilidad no tiene una especificación econométrica, y muy posiblemente los estimadores que produce no pasarían pruebas de sesgo (ya que no es este el objeto central de la investigación); es de esperarse que cualquier refinamiento que se hiciera en este sentido tenderá a mejorar la precisión de los resultados hasta ahora obtenidos.

\section{Bibliografía}

Barone-Adesi, G. and R.E. Whaley (1987). Efficient analytic approximation of American option values. Journal of Finance, 42, pp. 301-320.

Barraquand, J. and D. Martineau (1995). Numerical valuation of high dimesional multivariate american securities. Journal of Financial and Quantitative Analysis, 30, pp. 383-405.

Black, F. and M. Scholes (1973). The pricing of options and corporate liabilities. Journal of Political Economy, 81, pp. 637-659.

Blomeyer, E.C. (1986). An analytic approximation for the American put price of options on stocks with dividends. Journal of Financial and Quantitative Analysis, 21, pp. 229-233.

Briys, E., M. Bellalah, H.M. Mai and F. de Varenne (1998). Options, Futures and Exotic Derivatives. Chichester, West Sussex: John Wiley \& Sons, Ltd.

Broadie, M. and P. Glasserman (1997). Pricing American-sytle securities using simulation. Journal of Economic Dynamics and Control, 21, pp. 1323-1352.

Broadie, M., P. Glasserman and G. Jain (1997). Enhanced Monte Carlo estimates for American option prices. Journal of Derivatives, 5, pp. 25-44.

Cox, J.C., S.A. Ross and M. Rubinstein (1979). Option Pricing: A Simplified Approach . Joumal of Financial Economics, 7, pp. 229-263.

Fundia, A. D. (2002). A Fast Monte Carlo Algorithm for Pricing American Options. Revista Mexicana de Economía y Finanzas, 1(3), pp. 243-253.

Geske, R. (1979a). The valuation of compound options. Journal of Financial Economics, 7 , pp. $63-81$.

Geske, R. (1979b). A note on the analytic formula for unprotected American call options on stocks with known dividends. Journal of Financial Economics, 7, pp. 375-380.

Geske, R. and H.E. Johnson (1984). The American put option valued analytically. Journal of Finance, 39, pp. 1511-1524.

Geske, R. and K. Shastri (1985). Valuation by approximation: a comparison of alternative option valuation techniques. Journal of Financial and Quantitative Analysis, 20, pp. 45-71.

Hull, J.C. (2002). Options, Futures, and Other Derivatives. $5^{\text {th }}$ ed. Upper Saddle River, New Jersey: Prentice-Hail.

Hull, J.C. and A. White (1988). The use of the Control Variate Technique in Option Pricing. Journal of Financial and Quantitative Analysis, 23, pp. 237-251.

Johnson, H.E. (1983). An analytic approximation to the American put price. Journal of Financiol and Quantitative Analysis, 18, pp, 141-148.

McMillan, L. (1986). Analytic approximation for the American put option. Advances in Futures and Options Research, 1, pp. 119-139.

Merton R.C. (1973). Theory of rational option pricing. Bell Journal of Economics and Management Science, 4, pp. 141-183. 
Roll, R. (1977). An analytic valuation formula for unprotected American call options on stocks with known dividends. Journal of Financial Economics, 5, pp. 251-258.

Tilley, J.A. (1993). Valuing American options in a path simulation model. Transactions of the Society of Actuaries, 45, pp. 83-104.

Whaley, R.E. (1981). On the valuation of American call options on stocks with known dividends. Journal of Financial Economics, 9, pp. 207-211.

Whaley, R.E. (1982). Valuation of American call options on dividend paying stocks: empirical tests. Journal of Financial Economics, 10, pp. 29-58.

Whaley, R.E. (1986). Valuation of American futures options: theory and empirical tests. Journal of Finance, 41, pp. 127-150. 